\title{
Carbohydrate deficient transferrin and alcoholism
}

\author{
Hilary Denis Solomons*
}

Abstract

Alcohol abuse is an important public health problem, with major implications in patients with a preexisting liver pathology of viral origin. Hepatitis $\mathrm{C}$, for example, is one of the diseases in which alcohol consumption can lead to the transition from a fairly benign outline to a potentially life-threatening liver disease.

Alcohol abuse is usually identified on the basis of clinical judgment, alcoholism related questionnaires, laboratory tests and, more recently, biomarkers. Also on this list of tests, carbohydrate deficient transferrin (CDT) is widely available and useful for determining recent alcohol consumption, particularly when corroborated with elevation of other liver-associated enzymes. Clinicians should be aware of the indications and limitations of this test in order to better evaluate alcohol consumption in their patients.

Keywords Carbohydrate deficient transferrin, hepatitis, alcohol consumption

Alcohol abuse is an important public health problem. This condition is usually identified on the basis of clinical judgment, alcoholism related questionnaires and laboratory tests, i.e. gammaglutamyl transferase (GGT), aspartate aminotransferase (AST) or mean cell volume (MCV). However, the relatively low sensitivity and specificity of these tests $(50 \%$ sensitivity and $96.7 \%$ specificity for GGT, $70 \%$ and $93.3 \%$ for AST and MCV) ${ }^{1}$ led to a search for a specific marker. Several tests have recently been proposed, such as mitochondrial acetaldehyde adducts, beta-hexosaminidase or phosphatidylethanol (PEth), with promising results.

Carbohydrate deficient transferrin (CDT) has been shown to be more useful than other widely available biochemical tests for alcohol abuse. Structurally, transferrin is a polypeptide with two $\mathrm{N}$-linked polysaccharide chains, branched with sialic acid residues. According to the level of

Received: 19 March 2012; accepted: 22 April 2012

${ }^{*}$ Corresponding author: Hilary Denis Solomons, MB BCh, M Med Hematology, Pathology, University of the Witwatersrand, P.O. Box 64203, Highlands North, 2037, South Africa; soljer@netactive.co.za

Article downloaded from www.germs.ro

Published on 1 June 2012

(C) GERMS 2012

ISSN $2248-2997$

ISSN - L = $2248-2997$ sialylation, there are various forms of transferrin, tetrasialotransferrin being predominant. The proportion of transferrin with zero, one or two sialic acid chains increases with alcohol consumption but also in the carbohydrate deficient glycoprotein syndrome, leading to the so-called carbohydrate deficient transferrin.

Elevated levels of CDT are suggestive of recent alcohol abuse, particularly when corroborated with elevation of other liverassociated enzymes. Some of the uses of CDT include long term monitoring for early detection of relapse drinking during medical treatment, permitting early intervention. ${ }^{2}$ It may also aid in the assessment for reinstating driver licenses or in identifying chronic alcoholics among traumatized patients. ${ }^{3}$

Methods of determination include: ionexchange chromatography on minicolumns, radioimmunoassay, immunoblotting, densitometry and high performance liquid chromatography (HPLC), which has been recommended as reference method until a standardized mass spectrometric method is established. ${ }^{4}$ Through HPLC, five transferrin isoforms are separated at $460 \mathrm{~nm}$ : asialo-, disialo-, trisialo-, tetrasialo- and pentasialo-transferrin isoforms. ${ }^{5}$ The clinical significance of CDT is determined by looking specifically at the disialo and asialo forms. Slight differences between 
testing methods may be related to the tests' ability to separate and detect asialo, monosialo, and disialo isoforms. ${ }^{6}$ The International Federation of Clinical Chemistry and Laboratory Medicine (IFCC) Working Group on CDT Standardization recommended disialotransferrin as the primary target molecule for CDT measurement and the single analyte for CDT standardization. ${ }^{4}$

CDT has been used as a test for excessive alcohol consumption in research, clinical, and medico-legal settings, but there still is conflicting data on its accuracy, with sensitivities ranging from under $20 \%$ up to $100 \% .{ }^{7}$ In field literature, the results of paired studies obtained with commercially available CDT assays did not prove to be significantly better than with GGT. Scouller et al., after performing a meta-analysis of the data published so far, concluded that further highquality studies comparing CDTect (modified) and other CDT assays with GGT in the same subjects are needed. ${ }^{7}$

A more recent study ${ }^{1}$ compared CDT, MCV, AST, ALT, GGT and sialic acid (SA) in subjects which had declared themselves recently abstinent from alcohol consumption. Their results showed that CDT appeared to have higher sensitivity (95\% compared with $70 \%$ for MCV and AST, $50 \%$ for ALT and GGT and only 10\% for SA) but the sensitivity decreased for all studied alcohol markers when the period of abstinence was longer than one week. ${ }^{1}$ In this context, it might be useful to also discuss the use of markers which yield a longer time horizon for judging drinking levels. For example, measurement of ethyl glucuronide $(\mathrm{EtG})$ from hair is increasingly used for determining chronically increased alcohol consumption. ${ }^{8,9}$ A study comparing hair $\mathrm{EtG}$ and CDT in subjects with a two-week and/or three-months history of $60 \mathrm{~g} /$ day ethanol intake showed superior sensitivity of EtG (100\% vs. $44 \%$ for CDT) with a similar specificity of $93 \%$ at two weeks, measured by immunonephelometry for cut-off values of $27 \mathrm{pg} / \mathrm{mg}$ for EtG and $2.5 \%$ for CDT. The same study reported different results when measurements were performed through HPLC: 96\% sensitivity for $\mathrm{EtG}$ vs. $50 \%$ for $\mathrm{CDT}$ and an equal specificity of $70 \% .^{10}$
One other remarkably good direct marker of alcohol intake is phosphatidylethanol (PEth) ${ }^{11}$ which can be detected in blood for up to two weeks of sobriety. ${ }^{12}$ Other studies focused on the sensitivity and specificity of ethanol and methanol concentration in plasma, and the 5hydroxy-tryptophol (5-HTOL) to 5-hydroxyindoleacetic acid (5-HIAA) ratio in urine as laboratory tests to identify acute alcohol consumption. Comparison was made with self reported drinking levels. The study included different drinking categories (nondrinkers, light/moderate drinkers, heavy drinkers, patients receiving treatment for alcohol dependence) from five study populations (Australia, Brazil, Canada, Finland and Japan) and it showed that 5HTOL/5-HIAA ratio was the most sensitive and ethanol the least sensitive indicator of recent alcohol consumption. ${ }^{13}$

Given the fact that transferrin is a steroidresponsive protein, CDT is known to vary with sex and possibly with age. ${ }^{14}$ Therefore, the IFCC Working Group proposed percent carbohydratedeficient transferrin (\%CDT) as preferred method of reporting relative to total $\mathrm{CDT}^{4}{ }^{4} \mathrm{~A}$ widely used clinical cutoff value for heavy drinking is over $2.6 \%{ }^{15}$

A limitation for the use of CDT resides in the fact that abnormal serum transferrin glycoforms (with different numbers of sialic acid residues) can be found in carbohydrate deficient glycoprotein syndromes. Iourin et al. ${ }^{16}$ confirm the presence of hexa-, penta- and tetra-sialoforms of human serum transferrin in both normal samples and in type I carbohydrate deficient glycoprotein syndrome, concluding that capillary zone electrophoresis can be used as rapid diagnostic test for carbohydrate deficient glycoprotein syndromes, ${ }^{16}$ data also confirmed through the research of Wuyts et al. ${ }^{17}$

Alcohol consumption can be a major issue in patients with a pre-existing liver pathology of viral origin. Hepatitis C, for example, is one of the diseases in which alcohol consumption can lead to the transition from a fairly benign outline to a potentially life-threatening liver disease. ${ }^{18,19} \mathrm{Wu}$ et al. hypothesized that nuclear receptor signaling is 
altered in patients with chronic hepatitis C, displaying a pattern specific to alcohol drinking history. ${ }^{20}$ Their study showed that, in chronic $\mathrm{HCV}$ infection of the liver, alcohol intake was correlated with an increased expression of genes involved in fatty acid uptake, trafficking and oxidation, and a decreased expression of gluconeogenesis genes. ${ }^{20}$

Osna et al. reported that alcohol-consuming patients with hepatitis $\mathrm{C}$ virus (HCV) frequently have a longer course of infection and more severe manifestations of chronic hepatitis, excessive ethanol consumption being known to synergize with HCV to exacerbate liver injury (through modulation of $\mathrm{HCV}$ replication by ethanol metabolism). ${ }^{21}$ In clinical practice, questionnaires are used for estimating the alcohol intake of patients. ${ }^{22}$ Given the fact that questionnaires are highly subjective, CDT has also been considered in ascertaining alcohol consumption in patients with viral hepatitis. However, since transferrin is synthesized, glycosylated, and secreted by the liver, the use of CDT in patients with liver disease can raise certain problems. For example, elevated CDT values may not accurately correlate with alcohol consumption in advanced liver disease while patients with hepatitis $\mathrm{C}$ have been shown to have a higher chance of having a clinically positive CDT compared with patients with other types of liver diseases. ${ }^{23}$

Perhaps we are reaching a stage where the CDT value as a percentage may even be a predictor of who would make a good candidate for liver transplant? A value greater than 6.5 for example. Not assuming that it will obviate liver function tests or histology of liver biopsy, the CDT value may however give one a good indication of impending liver failure and the need for a transplant.

In conclusion, CDT is a marker of chronic alcohol abuse and current assays can be used in detecting an alcohol consumption of 40-60 g of ethanol per day, consumed for 2-3 weeks or longer. ${ }^{24}$ It has been proven to be superior to GGT or MCV as an indicator of chronic alcohol or ethanol abuse. ${ }^{1}$ It is a sensitive $(95 \%)$ and specific $(93.3 \%)$ marker of chronic imbibing ${ }^{1}$ and alcoholic hepatitis, if not liver cirrhosis (micronodular and macronodular cirrhosis). It may also be a marker of liver or hepatic failure. Liver function tests (e.g., transaminases) may well be elevated and liver biopsies will show steatosis, Mallory bodies and evidence of fatty infiltration while carbohydrate deficient or depleted transferrin is indeed of value in the detection of chronic alcohol abuse.

References

1. Chrostek L, Cylwik B, Szmitkowski M, Korcz W. The diagnostic accuracy of carbohydrate-deficient transferrin, sialic acid and commonly used markers of alcohol abuse during abstinence. Clin Chim Acta. 2006; 364(1-2):167-71.

2. Ciobanu A. Efficacy of trazodone in relapse prevention on alcohol dependence. Therapeutics, Pharmacology and Clinical Toxicology. 2006; X(1).

3. Huseby NE, Nilssen O, Erfurth A, Wetterling T, Kanitz RD. Carbohydrate-deficient transferrin and alcohol dependency: variation in response to alcohol intake among different groups of patients. Alcohol Clin Exp Res. 1997; 21(2):201-5.

4. Jeppsson JO, Arndt T, Schellenberg F, Wielders JP, Anton RF, Whitfield JB, et al. Toward standardization of carbohydrate-deficient transferrin (CDT) measurements: I. Analyte definition and proposal of a candidate reference method. Clin Chem Lab Med. 2007; 45(4):558-62.

5. Lesch OM, Walter H, Freitag H, Heggli DE, Leitner A, Mader R, et al. Carbohydrate-deficient transferrin as a screening marker for drinking in a general hospital population. Alcohol Alcohol. 1996; 31(3):249-56.

6. Sillanaukee P, Lof K, Harlin A, Martensson O, Brandt R, Seppa K. Comparison of different methods for detecting carbohydrate-deficient transferrin. Alcohol Clin Exp Res. 1994; 18(5):1150-5.

7. Scouller K, Conigrave KM, Macaskill P, Irwig L, Whitfield JB. Should we use carbohydrate-deficient transferrin instead of gamma-glutamyltransferase for detecting problem drinkers? A systematic review and metaanalysis. Clin Chem. 2000; 46(12):1894-902.

8. Yegles M, Labarthe A, Auwarter V, Hartwig S, Vater $\mathrm{H}$, Wennig R, et al. Comparison of ethyl glucuronide and fatty acid ethyl ester concentrations in hair of alcoholics, social drinkers and teetotallers. Forensic Sci Int. 2004; 145(2-3):167-73.

9. Kronstrand R, Brinkhagen L, Nystrom FH. Ethyl glucuronide in human hair after daily consumption of 16 or $32 \mathrm{~g}$ of ethanol for 3 months. Forensic Sci Int. 2012; 215(1-3):51-5.

10. Morini L, Politi L, Acito S, Groppi A, Polettini A. Comparison of ethyl glucuronide in hair with carbohydrate-deficient transferrin in serum as markers 
of chronic high levels of alcohol consumption. Forensic Sci Int. 2009; 188(1-3):140-3.

11. Lundqvist C, Aradottir S, Alling C, Boyano-Adanez MC, Gustavsson L. Phosphatidylethanol formation and degradation in brains of acutely and repeatedly ethanol-treated rats. Neurosci Lett. 1994; 179(1-2):12731.

12. Bendroth P, Kronstrand R, Helander A, Greby J, Stephanson N, Krantz P. Comparison of ethyl glucuronide in hair with phosphatidylethanol in whole blood as post-mortem markers of alcohol abuse. Forensic Sci Int. 2008; 176(1):76-81.

13. Helander A, Eriksson CJ. Laboratory tests for acute alcohol consumption: results of the WHO/ISBRA Study on State and Trait Markers of Alcohol Use and Dependence. Alcohol Clin Exp Res. 2002; 26(7):10707 .

14. Das S, Vasudevan D. Should we use carbohydrate deficient transferrin as a marker for alcohol abusers? Indian J Clin Biochem. 2004; 19(2):36-44.

15. Marques PR. Levels and types of alcohol biomarkers in DUI and clinic samples for estimating workplace alcohol problems. Drug Test Anal. 2012; 4(2):76-82.

16. Iourin O, Mattu TS, Mian N, Keir G, Winchester B, Dwek RA, et al. The identification of abnormal glycoforms of serum transferrin in carbohydrate deficient glycoprotein syndrome type I by capillary zone electrophoresis. Glycoconj J. 1996; 13(6):103142.

17. Wuyts B, Delanghe J, Kasvosve I, Wauters A, Neels H, Janssens J. Determination of carbohydrate-deficient transferrin using capillary zone electrophoresis. Clinical Chemistry. 2001; 47(2):247-55.

18. Vento S, Cainelli F. Does hepatitis C virus cause severe liver disease only in people who drink alcohol? Lancet Infect Dis. 2002; 2(5):303-9.

19. Cui Q, Zhang Y, Su J, et al. The association between the genetic polymorphisms of LMP2/LMP7 and the outcomes of $\mathrm{HCV}$ infection among drug users. J Biomed Res. 2010;24(5):374-80.

20. Wu C, Gilroy R, Taylor R, Olyaee M, Abdulkarim B, Forster J, et al. Alteration of hepatic nuclear receptormediated signaling pathways in hepatitis $\mathrm{C}$ virus patients with and without a history of alcohol drinking. Hepatology. 2011; 54(6):1966-74.

21. Osna NA, Thomes PG, Jr TM. Involvement of autophagy in alcoholic liver injury and hepatitis C pathogenesis. World J Gastroenterol. 2011; 17(20):2507-14.

22. Streinu-Cercel A, Rebedea I, Streinu-Cercel O, Streinu-Cercel A, Preoțescu L, Manolache D. Hepatitis $C$ virus superinfection in individuals with chronic hepatitis B virus infection. A comparative case study. Therapeutics, Pharmacology and Clinical Toxicology. 2010; XIV(1):25-35.

23. DiMartini A, Day N, Lane T, Beisler AT, Dew MA, Anton R. Carbohydrate deficient transferrin in abstaining patients with end-stage liver disease. Alcohol Clin Exp Res. 2001; 25(12):1729-33.

24. Golka K, Wiese A. Carbohydrate-deficient transferrin (CDT)-a biomarker for long-term alcohol consumption. J Toxicol Environ Health B Crit Rev. 2004; 7(4):319-37.

Please cite this article as:

Solomons HD. Carbohydrate deficient transferrin and alcoholism. GERMS. 2012;2(2):75-8 